\title{
Custo-minimização de curativos para cateter venoso central: gaze estéril versus filme transparente
}

\author{
Cost-minimization of bandage for central venous catheter: sterile gauze versus transparent film
}

Minimización de costes del vendage para catéter venoso central: gasa estéril versus película transparente

\author{
Fernanda Oliveira Caló', Antônio Augusto de Freitas Peregrino", Andrezza Serpa Franco'", \\ Ronilson Gonçalves Rocha ${ }^{\text {IV }}$, Flavia Giron Cameriniv ${ }^{\text {, Cristiano Bertolossi Marta }}{ }^{\text {VI }}$
}

\begin{abstract}
RESUMO
Objetivo: comparar o custo entre coberturas com filme transparente versus gaze estéril e fita adesiva microporosa em curativos de acesso venoso central. Método: estudo estatístico, prospectivo, observacional, com abordagem quantitativa à luz da avaliação econômica de saúde. Foram avaliadas 109 coberturas com filme e $168 \mathrm{com}$ gaze, durante 27 dias. Na análise utilizouse o Software Tree Age para se calcular a probabilidade de cada decisão. Resultado: a média de uso das coberturas por dia foi de 2,22 inserções cobertas com filme e 3,43 com gaze. Houve necessidade de troca extra em 38 (23\%) das coberturas com gaze e em $16(15 \%)$ das coberturas com filme transparente. Observou-se custo final médio para gaze $(R \$ 389,44) 3,7$ vezes maior que o filme transparente $(R \$ 104,95)$ durante o período estudado. Conclusão: cobertura com filme transparente tem menor custo quando comparado com cobertura com gaze estéril.
\end{abstract}

Descritores: Avaliação em Saúde; Custos e Análise de Custo; Infecções Relacionadas a Cateter.

\section{ABSTRACT}

Objective: to compare the cost of transparent film and sterile gauze fixed by microporous adhesive tape in central venous access dressings. Method: quantitative, prospective, observational, statistical study in the light of health economics. A total of 109 film and 168 gauze covers were evaluated for 27 days. Tree Age software was used to calculate the probability of each decision. Results: coverings used per day averaged 2.22 with transparent film-covered inserts and 3.43 with gauze. Additional changes were necessary in $38(23 \%)$ coverings with gauze and $16(15 \%)$ with transparent film. During the study period, the average final cost of gauze ( $R \$ 389.44)$ was 3.7 times higher than transparent film ( $R \$ 104.95)$. Conclusion: transparent film dressing is less expensive than sterile gauze covering.

Descriptors: Health Evaluation; Costs and Cost Analysis; Catheter-Related Infections.

\section{RESUMEN}

Objetivo: comparar el costo de la película transparente y la gasa estéril fijada con cinta adhesiva microporosa en apósitos de acceso venoso central. Método: estudio cuantitativo, prospectivo, observacional, estadístico a la luz de la economía de la salud. Se evaluaron un total de 109 películas y 168 cubiertas de gasa durante 27 días. Se utilizó el software Tree Age para calcular la probabilidad de cada decisión. Resultados: los recubrimientos utilizados por día promediaron 2,22 con inserciones transparentes recubiertas con película y 3,43 con gasas. Fueron necesarios cambios adicionales en $38(23 \%)$ revestimientos con gasa y $16(15 \%)$ con película transparente. Durante el período de estudio, el costo final promedio de la gasa $(R \$ 389,44)$ fue 3,7 veces superior al de la película transparente $(\mathrm{R} \$ 104,95)$. Conclusión: el apósito de película transparente es menos costoso que el recubrimiento de gasa estéril.

Descriptores: Evaluación en Salud; Costos y Análisis de Costo; Infecciones Relacionadas con Catéteres.

\section{INTRODUÇÃO}

Esse estudo foi desenvolvido a partir de inquietações de enfermeiros assistenciais sobre a melhor opção de coberturas para cateter venosos sobre a perspectiva de custos. Nesse sentido foi realizada uma avaliação comparativa entre a utilização de gaze estéril e película transparente em curativo de acesso venoso central, através de uma análise de custo minimização sob a ótica de prevenção de infecção.

As Infecções Relacionadas à Assistência de Saúde (IRAS) se referem às infecções adquiridas e relacionadas aos cuidados de saúde em qualquer tipo de ambiente. Dentre as mais frequentes em pacientes críticos, destacam-se as Infecções de Corrente Sanguínea (ICS) ${ }^{1}$.

'Enfermeira. Universidade do Estado do Rio de Janeiro. Rio de Janeiro, Brasil. E-mail: fcalo.enfermeira@gmail.com. ORCID: http://orcid.org/0000-0002-6735-8109 "Enfermeiro. Pós-Doutorado. Universidade do Estado do Rio de Janeiro. Rio de Janeiro, Brasil. E-mail: antonio.peregrino@gmail.com. ORCID: http://orcid.org/0000-0002-6617-480X I'Enfermeira. Doutora. Universidade do Estado do Rio de Janeiro. Rio de Janeiro, Brasil. E-mail: dezzafranco@gmail.com. ORCID: http://orcid.org/0000-0001-5008-1345 IVEnfermeiro. Doutor. Universidade do Estado do Rio de Janeiro. Rio de Janeiro, Brasil. E-mail: ronilsonprof@gmail.com. ORCID: http://orcid.org/0000-0003-4097-8786 VEnfermeira. Doutora. Universidade do Estado do Rio de Janeiro. Rio de Janeiro, Brasil. E-mail: fcamerini@gmail.com. ORCID: http://orcid.org/0000-0002-4330-953X VIEnfermeiro. Pós-Doutorado. Universidade do Estado do Rio de Janeiro. Rio de Janeiro, Brasil. E-mail: cristianobertol2014@gmail.com. ORCID: http://orcid.org/0000-0002-0635-7970 
A mortalidade por ICS é variável entre pacientes e relaciona-se diretamente com outros fatores de risco, como por exemplo, o tempo de permanência em Terapia Intensiva. A Agência Nacional de Vigilância Sanitária (ANVISA) cita resultados oriundos de notificação compulsória com a densidade de incidência de ICS em hospitais brasileiros, que mostraram 18.370 casos por ano de ICS, das quais $60 \%$ ocorreram em unidades de Terapia Intensiva adulta ${ }^{1}$.

Entende-se que as ICS são multifatoriais e dependem de uma série de condições para nortear diagnóstico e tratamento adequado. Na prática, se subdividem em: infecções primárias de corrente sanguínea (IPCS) e infecções relacionadas ao acesso vascular (IAV), que apresentam quadro de prevenção e diagnósticos específicos e distintos. As IAV apesar de mais frequentes, são consideradas de menor gravidade e possuem sinais de infecção visíveis no local de inserção do cateter como, por exemplo, secreção purulenta e hiperemia².

Apesar do uso de cateter venoso central (CVC) ser amplamente utilizado e apresentar benefícios indiscutíveis aos pacientes críticos, o uso desse tipo de dispositivo pode aumentar o risco de complicações, como a ICS ${ }^{3}$. A ICS apresenta uma enorme preocupação, pois é considerada a infecção associada a cuidados em saúde de maior potencial preventivo que existe. Estudos estimam que cerca de $60-75 \%$ dos casos poderiam ser prevenidos, através da otimização das práticas de manutenção e manipulação dos dispositivos vasculares ${ }^{1,4}$.

A manutenção do uso de CVC requer uso de cobertura apropriada, que tem o propósito de proteger o sítio de punção e minimizar a possibilidade de infecção por meio da interface entre a superfície do cateter e a pele, além de estabilizar o dispositivo no local e prevenir a movimentação do dispositivo com potencial dano ao vaso. Entende-se que a cobertura ideal, deve manter-se oclusiva, seca e estéril para a proteção do óstio de inserção do cateter. Para tal, a ANVISA sugere o uso de cobertura com gaze estéril e fixador ou com película transparente semipermeável estéril, ambas com nível moderado de evidência².

Em várias unidades hospitalares, o método atual de cuidados com CVC inclui curativos oclusivos diários utilizando técnica asséptica com gaze e luvas estéreis, fita adesiva microporosa e solução alcoólica de clorohexidina a 0,5\% ou, na falta deste, álcool 70\%. Alternativas como uso de filme transparente estéril substituiriam a manipulação diária do sítio de inserção, porém envolvem a manipulação correta da cobertura, habilidade e treinamento da equipe ${ }^{5}$.

$\mathrm{Na}$ avaliação da efetividade na prevenção de IAV em CVC, um estudo apontou probabilidades semelhantes de ocorrência de infecção de óstio, sendo $4 \%$ para coberturas com gaze e 3\% no uso do filme estéril. Uma diferença estatisticamente pouco significativa, ou seja, as coberturas se mostraram equivalentes com relação a prevenção de infecção de corrente sanguínea. Diante disso, esse estudo tem por base analisar os custos envolvidos entre os dois métodos de proteção do óstio de $\mathrm{CVC}^{6}$.

Avaliações do tipo custo-minimização consideram a efetividade das intervenções comparadas como equivalentes, sendo o foco da análise os custos envolvidos em cada uma delas, indicando aquela que tem menor custo ${ }^{7}$.

Estudos dessa natureza são pouco frequentes no âmbito da enfermagem, embora a categoria possua grande representatividade nos serviços de saúde. A enfermagem tem atuação no processo de compra e solicitação de novos materiais, assim como sua avaliação, o que influencia no aspecto econômico, devido ao poder de decisão em determinar o destino dos recursos, além de conhecer a fundo as necessidades assistenciais do setor que trabalham. Somado a isso, tem-se o conceito que as ICS aumentam a mortalidade, o tempo de internação e custos de hospitalização, sendo, portanto, uma questão de relevância na gestão de recursos financeiros ${ }^{8}$.

A questão de estudo dessa pesquisa é: "Qual o custo-minimização da realização de curativo com filmes transparentes estéril, comparados aos curativos estéreis diários com gaze estéril fixada com fita adesiva microporosa em acesso venoso central de pacientes adultos de unidades de terapia intensiva, no âmbito de um hospital universitário, durante período de uso do dispositivo?".

Utilizou-se a hipótese de que o uso de curativos estéril com filme transparente pode promover a diminuição do gasto de materiais, além de reduzir o tempo de trabalho da equipe de enfermagem em pacientes em uso de acessos vasculares em unidades de terapia intensiva, uma vez que a necessidade de troca é menor comparada com a gaze estéril fixada com fita adesiva.

Frente ao exposto, teve-se como objetivo, comparar o custo entre coberturas com filme transparente versus gaze estéril e fita adesiva microporosa em curativos de acesso venoso central.

\section{MÉTODO}

Estudo estatístico, prospectivo, observacional, com abordagem quantitativa à luz da avaliação econômica em saúde. Está registrado na Plataforma Brasil e foi aprovado pelo Comitê de Ética em Pesquisa da instituição em 08 de março de 2017, pelo Parecer Consubstanciado no 1.953.828. Houve autorização de dispensa do uso do Termo de Consentimento Livre e Esclarecido. 
Foram coletados dados relacionados a coberturas e avaliação de óstio de inserção de CVC em unidade de terapia intensiva adulta em um hospital universitário. O local de pesquisa possui capacidade para dez leitos. Utilizou-se o Protocolo Operacional Padrão (POP) da unidade para confecção do check list da prática estudada. Organizou-se com a equipe de enfermagem que os leitos de um ao cinco utilizariam a cobertura com filme transparente estéril e os leitos de seis a dez, a cobertura com gaze estéril fixada com fita adesiva microporosa. O filme transparente faz parte da lista de materiais presentes na unidade, porém é utilizado com menor frequência devido ao seu alto custo inicial. Para realização desse estudo, a chefia da unidade direcionou a quantidade existente para uso na pesquisa.

Foram incluídas coberturas de CVC com filme transparente e com gaze estéril fixada com fita adesiva microporosa; coberturas de CVC inserido em veia jugular interna ou veia subclávia dos pacientes internados na unidade de terapia intensiva adulta estudada devido à possibilidade de acompanhamento diário e de sua frequência de instalação na unidade. Foram excluídos cateteres para hemodiálise, devido à dificuldade de acompanhamento das trocas diárias e da padronização da equipe, pois são realizados pela equipe específica da nefrologia; e o cateter periférico devido a não utilização de filme transparente estéril em seu curativo. Não se utilizaram cateteres inseridos por veia femural devido ao aumento da possibilidade de troca na região, por motivos que estão fora do escopo do estudo, podendo gerar um confundimento no resultado.

A coleta de dados ocorreu entre os meses de abril e julho de 2017. Inicialmente, apresentou-se a proposta às equipes de enfermagem de cada plantão ressaltando os objetivos do estudo, método de coleta, forma de aplicação do filme, correta avaliação da manutenção e da importância dos registros em prontuário. A pesquisadora acompanhou a troca diária dos curativos realizados com gaze estéril e com filme transparente estéril. Foram realizadas 49 coletas, em 87 dias diferentes (14 semanas). Em cada coleta eram observados quantos pacientes estavam em uso de cada cobertura, as características de cada uma e os registros do prontuário relacionado ao cateter e ao curativo, naquele determinado momento.

O registro das características das coberturas e avaliação do óstio foi feito utilizando check-list criado e preenchido pela pesquisadora. Este check-list objetivou quantificar número de pacientes em uso de cada cobertura e, dentre cada cobertura, quantas estavam adequadas (limpa/seca, oclusiva e data adequada), e inadequadas com alguma condição que determinava a realização de novo curativo (soltura, umidade/sujidade ou data inadequada/ausente), sinais de infecção local e espaço para observações extras. Os sinais de infecção eram visualizados diretamente nas coberturas com filme ou identificados indiretamente por meio dos registros de enfermagem no prontuário dos pacientes em uso de coberturas com gaze.

O instrumento permitia até quatro coletas diárias, durante serviço diurno (SD). O SD foi escolhido por caracterizarse como período de grande atividade de manipulação e/ou mobilização do paciente por profissionais da equipe de saúde, refletindo a efetividade da cobertura diante dos procedimentos diários.

Os dados coletados foram inseridos em planilha do Microsoft Office Excel $2010^{\circ}$ e separados segundo tipo de cobertura e características observadas. Após, foram realizados cálculos descritivos de tendência central, probabilidade e desvio-padrão por característica observada.

Para o cálculo de custos foi utilizada técnica de microcusteio, incluindo: curativo com gaze estéril, curativo com filme transparente, retirada de CVC, punção de novo CVC. Para o levantamento dos custos de materiais necessários utilizou-se informações do protocolo usado na unidade, tabela recente de compras do hospital e valor pago pela tabela de remuneração do Sistema Único de Saúde. O curativo utiliza os seguintes materiais: álcool glicerinado, luvas de procedimento e estéreis, flaconetes de soro fisiológico 0,9\%, gaze estéril, clorexidina alcoólica $0,5 \%$, gorro, máscara cirúrgica, óculos de proteção e a cobertura escolhida ${ }^{9,10}$.

Considerando a variabilidade entre cada paciente o tempo gasto nos procedimentos foi cronometrado em ocasiões randômicas, para determinar a média de tempo gasto e seu desvio padrão, de forma a refletir melhor a realidade do setor e a variação dos custos.

Os custos relacionados ao tempo profissional, em minutos, foram calculados utilizando a média de valor salarial e carga horária de cada categoria envolvida (médica ou enfermagem) daqueles profissionais que possuem vínculo empregatício no serviço público, utilizando dados da Secretaria da Fazenda e Planejamento do Rio de Janeiro. A probabilidade da ocorrência de infecção em cada cobertura, foi inserida utilizando-se como base a probabilidade publicada em um estudo já concluído ${ }^{6}$.

Os resultados dos cálculos estatísticos e custos envolvidos foram inseridos na árvore de decisão utilizando Software Tree Age ${ }^{\circledast}$. A variável "soltura" da cobertura com filme foi retirada, pois não foram observadas ocorrências de cobertura solta durante as coletas.

A necessidade de realização de novo curativo (maior que um), a cada 24 horas para a gaze e a cada sete dias para o filme, foram consideradas como troca extra. 
Os custos com coberturas inadequadas foram inseridos na árvore considerando a soma do custo de um curativo (com gaze ou filme) mais o custo do curativo diário (no caso do filme, este valor era dividido por sete). No ramo "coberturas adequadas" foram inseridos apenas os custos de curativo diário.

\section{RESULTADOS}

Utilizando as probabilidades mencionada na sessão método e considerando o número de coberturas observadas, foi calculado o número provável de retirada de cateter e punção de novo CVC e inserido no cálculo de custos da árvore de decisão do Software Tree Age ${ }^{\oplus}$, demonstrado na Figura 1.

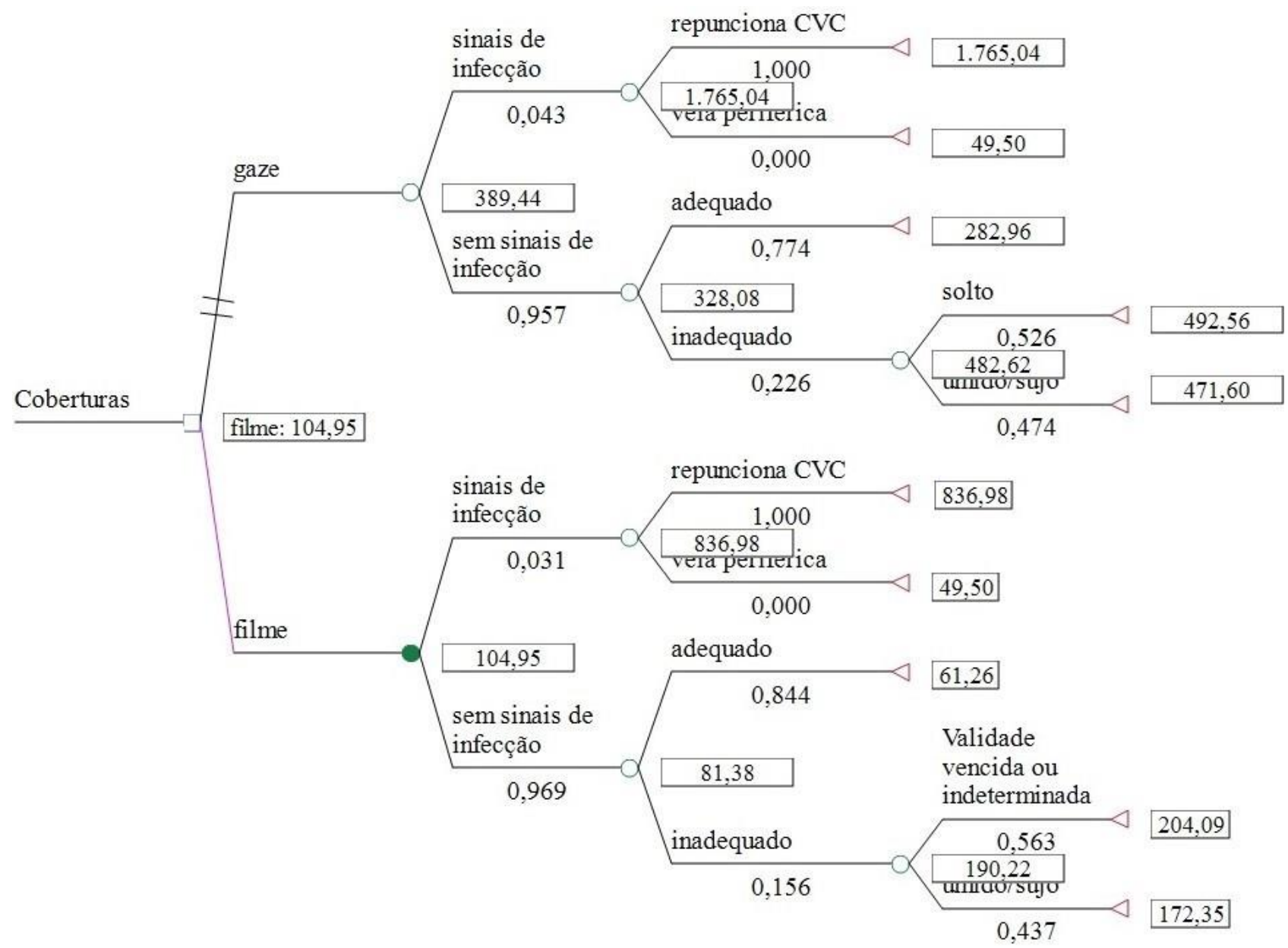

FIGURA 1: Árvore de decisão, com probabilidade e custos de cada ramo incluídos. Rio de Janeiro, Brasil, 2018.

No total, foram observadas 168 coberturas com gaze e 109 coberturas com filme. A média de uso das coberturas por dia foi de 2,22 inserções de CVC cobertas com filme e 3,43 com gaze.

Houve necessidade de troca extra, em 38 (23\%) coberturas com gaze, sendo 20 (12\%) devido a não oclusão total (curativos soltos) e 18 (11\%) por umidade/presença de sujidade. Foram identificadas dez trocas da cobertura, sem justificativa registrada.

Já nas coberturas com filme transparente, houve a necessidade de troca em 16 (15\%) curativos. Destas, a ausência de data de aplicação ou permanência maior que sete dias, representou um percentual de 9 (8\%), seguido por presença de umidade/sujidade em óstio em 7 (6\%) curativos.

A média semanal de trocas extras de coberturas com gaze foi de 9,5 e 4 para o filme transparente, essa média foi calculada dividindo o número total coberturas inadequadas pelo tempo de permanecia do cateter em semanas. 
O custo obtido para o procedimento de curativo foi de $\mathrm{R} \$ 10,48$ para cobertura com gaze e $\mathrm{R} \$ 15,87$ para filme. Estes custos refletem a relação dos custos envolvidos em cada cobertura (considerando as variações de tempo profissional envolvido que podem ocorrer em cada paciente, necessidade de trocas extras e necessidade de punção de novo CVC devido ocorrência de infecção) com a probabilidade envolvida em cada decisão.

Quando esses dados foram inseridos no Software Tree Age ${ }^{\circledR}$, foram obtidos dados numéricos dos custos envolvidos em cada cobertura no período, apresentados na Figura 2.

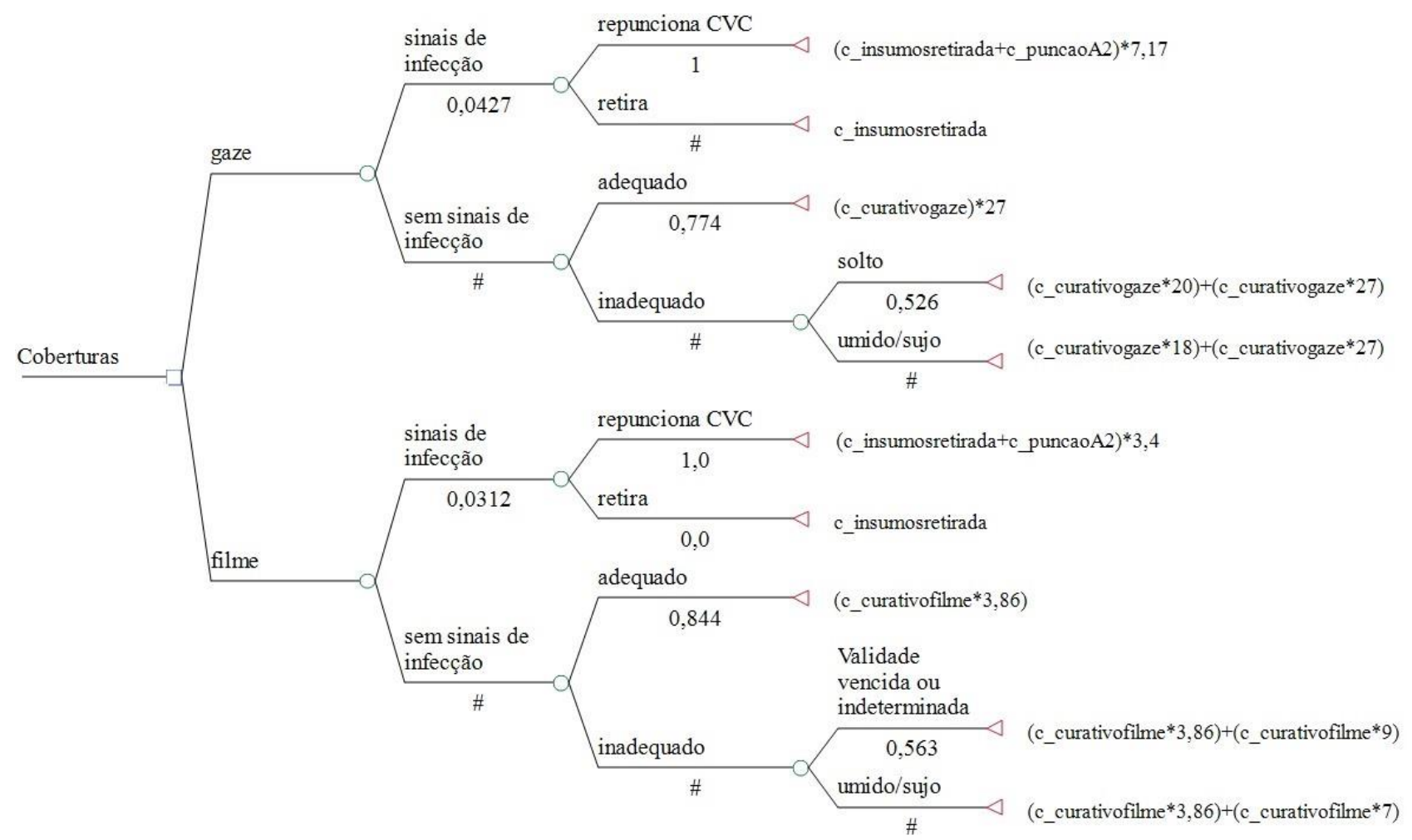

FIGURA 2: Árvore de decisão com custo x probabilidade de cada decisão. Rio de Janeiro, Brasil, 2018.

\section{DISCUSSÃO}

Aplicar ferramentas estatísticas que permitam utilizar dados contidos na literatura indexada e coletados in loco, permitem integrar e discutir melhor através da perspectiva econômica, contribuindo para replicar os achados em diferentes cenários. Na prática, o aumento de custos entre uma tecnologia e outra exige conhecimento para uma melhor gestão de materiais utilizados no hospital ${ }^{7,11}$.

Em um estudo publicado em 2015, foi avaliada a durabilidade e os custos relacionados às coberturas com filmes de diversas marcas em 590 cateteres e demonstrou que $28 \%$ dos filmes que necessitavam de troca foi devido à perda da aderência na pele. Tais resultados diferem dos dados coletados, em que não foi visualizada nenhuma cobertura com filme solta ${ }^{12}$.

Em uma etapa realizada previamente a coleta de dados, foi explicado aos profissionais, a importância de evitar tencionar o filme durante a aplicação, e de se certificar que a pele do paciente esteja limpa e seca antes de aplicar a cobertura de filme transparente. Há autores que defendem que a perda da aderência dessa tecnologia pode estar mais relacionada com a falta de capacitação da equipe, o que pode levar ao aumento da frequência de troca e a diminuição do seu custo-efetividade ${ }^{10,12}$.

No tocante às coberturas com gaze, um ensaio randomizado demonstrou uma probabilidade igual a 0,1 de não aderência das coberturas com gaze. No presente estudo, a probabilidade de não aderência da fixação de gaze com fita microporosa foi de $0,12 \%$ corroborando com os achados do ensaio. Destaca-se que o local de punção do CVC influencia 
fortemente na fixação do curativo e que os cateteres posicionados em veia jugular, tem maior chance de não aderência da cobertura, seja qual for o tipo de material ${ }^{10,13-14}$.

Economicamente, à primeira vista o filme transparente pode ser visto com ressalvas quanto aos benefícios financeiros devido à diferença do preço entre a unidade e o pacote com gaze estéril. Enquanto a cobertura com gaze custa $R \$ 0,39$, a unidade de filme transparente custa $R \$ 5,50$. Porém, ao considerar os sete dias de permanência ocorre uma inversão da diferença. O custo do curativo com gaze (levando em consideração o uso da luva estéril e da fita microporosa) passa a ser de $\mathrm{R} \$ 73,34$ enquanto que o gasto com o filme permanece (considerando o filme transparente e a luva estéril) em $\mathrm{R} \$ 15,87$ semanais. Considerando a média semanal de 4 trocas extras de coberturas com filme e 9,5 com gaze, acrescidas ao número de trocas mínimas necessárias, temos um gasto semanal médio de $\mathrm{R} \$ 79,35$ com o filme e de $\mathrm{R} \$ 177,92$ com a gaze.

Uma vez que as informações de custos e probabilidades foram inseridas na árvore de decisão e posteriormente calculados os valores relacionados a cada intervenção pode-se observar que o custo do uso de gaze estéril, durante período de coleta foi de $\mathrm{R} \$ 389,44$, um valor 3,7 vezes maior que o filme, que teve um custo de $\mathrm{R} \$ 104,95$ para um período de 30 dias. Esses valores aumentam progressivamente e para ilustrar o quanto essa progressão pode chegar pode-se fazer um cálculo simples.

Ao se realizar uma projeção para um ano haveriam, no mínimo, 365 trocas de cobertura com gaze e 52,14 trocas de filme. Se multiplicar esses números de trocas pelo valor do curativo com cada cobertura tem-se que o curativo com filme custaria $R \$ 827,46$ e com gaze custaria $R \$ 3825,20$. Um valor 4,62 vezes maior, sem ainda considerar a necessidade de trocas extras. Quando se insere esses valores em cenários nacionais dentro das perspectivas do SUS, pode-se chegar a números financeiros ainda maiores, que se poderia economizar e direcionar para outras práticas ${ }^{10,15}$.

Desta forma, percebe-se que a avaliação de custos de determinada prática nos apresenta a outras realidades, antes não vista. Fica evidente que, o uso de filme transparente num intervalo de tempo médio, pode gerar redução dos custos com a cobertura de CVC, através da redução do uso de materiais e da redução da exposição e manipulação do óstio, práticas que previnem a ocorrência de infecções de corrente sanguínea, e consequentemente, favorecem a redução do tempo de internação e seus custos associados.

\section{Limitações do estudo}

O estudo apresentou algumas limitações quanto a existência e a qualidade dos registros dos profissionais no prontuário em relação a execução dos curativos, embora haja no setor uma Sistematização da Assistência de Enfermagem (SAE) para registro das características do curativo. Além disso, houve uma limitação na sequência diária de observações das execuções dos curativos pela pesquisadora devido a restrição de acesso ao referido hospital devido à greve.

\section{CONCLUSÃO}

Após análise dos dados obtidos, foi possível comparar o custo do uso de curativos de CVC entre coberturas com filme transparente e com gaze estéril fixada e comprovar a hipótese inicial de que o filme tem menor custo quando comparado com a gaze. Além disso, mantem-se adequado por mais tempo o que reflete em um menor tempo de assistência do enfermeiro.

Os ensaios clínicos mostraram que a probabilidade de ocorrência de IPCS é ligeiramente menor no uso do filme, porém sem relevância estatística. Entretanto, é importante salientar que a possibilidade de visualização do óstio sem necessidade de retirar a cobertura, possibilita intervenções em menos tempo. Nesse estudo, houve identificação de apenas um óstio com sinais de infecção local em um curativo feito com gaze estéril. Parte desse resultado foi devido à dificuldade em obter dados em prontuário de forma fidedigna.

Os resultados deste estudo demonstram a importância da avaliação econômica na área de enfermagem, mesmo nas atividades mais cotidianas. Um olhar crítico possibilita aos profissionais repensarem seus conceitos e ações no sentido financeiro associado com melhora da assistência e do processo de trabalho. Essa análise não deve basear-se em pressões externas para se ter uma nova tecnologia, ou mesmo na resistência em adaptar-se às novidades, mas na busca pelo melhor resultado com menor custo, viabilizando e evoluindo sistema de saúde como um todo.

Diante disso, sugere-se a replicação desse estudo em outras unidades, que utilizam tais produtos para realização de coberturas de curativos de Cateter Venoso Central, a fim de ampliar a análise dos estudos sobre custos e as evidências científicas sobre a temática, auxiliando no processo de tomada de decisão para incorporação de determinada tecnologia. 


\section{REFERÊNCIAS}

1. Agência Nacional de Vigilância Sanitária. Medidas de prevenção de Infecção Relacionada à Assistência à Saúde. Segurança do Paciente e Qualidade em Serviços de Saúde. 2017 [cited 2020 Jun 6]. Available from:

http://portal.anvisa.gov.br/documents/33852/3507912/Caderno+4+-

+Medidas+de+Preven\%C3\%A7\%C3\%A3o+de+Infec\%C3\%A7\%C3\%A3o+Relacionada+\%C3\%A0+Assist\%C3\%AAncia+\%C3\%A0+Sa\% C3\%BAde/a3f23dfb-2c54-4e64-881c-fccf9220c373

2. Agência Nacional de Vigilância Sanitária. Critérios Diagnósticos de Infecção Relacionada à Assistência à Saúde. Segurança do Paciente e Qualidade em Serviços de Saúde. Brasília: Agência Nacional de Vigilância Sanitária; 2013 [cited 2020 Jun 6]. Available from: http://portal.anvisa.gov.br/documents/33852/3507912/Caderno+2+-

+Crit\%C3\%A9rios+Diagn\%C3\%B3sticos+de+Infec\%C3\%A7\%C3\%A3o+Relacionada+\%C3\%A0+Assist\%C3\%AAncia+\%C3\%A0+Sa\%C 3\%BAde/7485b45a-074f-4b34-8868-61f1e5724501

3. Mendonça KM, Neves HCC, Barbosa DFS, Souza ACS, Tipple AFV, Prado MA. Nursing care in the prevention and control of catheter-related bloodstream infections. Rev. enferm. UERJ. [Internet], 2011 [cited 2020 Jun 5]; 19(2):330-3. Available from: http://www.revenf.bvs.br/pdf/reuerj/v19n2/v19n2a26.pdf

4. Andrade AM, Borges KS, Lima HO. Cost analysis and evaluation of covers fora central venous catheter insertionsite in BMT. REME Rev. Min. Enferm. [Internet], 2011 [cited 2020 Aug 17]; 15(2):233-41. Available from: https://cdn.publisher.gn1.link/reme.org.br/pdf/v15n2a11.pdf

5. Agência Nacional de Vigilância Sanitária. Medidas de prevenção de Infecção Relacionada à Assistência à Saúde. Segurança do Paciente e Qualidade em Serviços de Saúde. 2017 [cited 2018 Jun 6]. Available from: http://portal.anvisa.gov.br/documents/33852/3507912/Caderno+4+-

+Medidas+de+Preven\%C3\%A7\%C3\%A30+de+Infec\%C3\%A7\%C3\%A3o+Relacionada+\%C3\%A0+Assist\%C3\%AAncia+\%C3\%A0+Sa\% C3\%BAde/a3f23dfb-2c54-4e64-881c-fccf9220c373

6. Pedrolo E. Custo-efetividade das coberturas para cateter venoso central de curta permanência. [Doctoral dissertation]. Curitiba: Universidade Federal do Paraná; 2017 [cited 2018 Jun 6]. Available from: https://acervodigital.ufpr.br/handle/1884/51269

7. Ministério da Saúde. Diretrizes metodológicas: Diretriz de Avaliação Econômica. Brasília: Ministério da Saúde; 2014 [cited 2018 Sep 27]. Available from: http://bvsms.saude.gov.br/bvs/publicacoes/diretrizes_metodologicas_diretriz_avaliacao_economica.pdf

8. Borgert $\mathrm{E}$, Gasparetto V. Factors that influence costs, in the approach of strategic cost management: empirical analysis in municipal health centers. Cuadernos de Contabilidad [Internet]. 2019 [cited 2020 jul 27]; 20(49):1-7. DOI: https://doi.org/10.11144/Javeriana.cc20-49.fica

9. Oliveira BCC, Silva JA, Pontes RL, Barbosa MGA, Silva EI, Silva BM, Lins SRO. The knowledge of the professionals of nursing about infections related to the use of central venous cateter of double lumen in dialytic patients in a nephrological clinic of the agreste of Pernambuco. Braz. J. of Develop. [Internet], 2020 [cited 2020 jul 27]; 6(7):44436-50. DOI: https://doi.org/10.34117/bjdv6n7-166

10. Paz A, Maciel R, Alves P, Souza RM. Elaboração do Procedimento Operacional Padrão de Enfermagem sobre Curativo de Cateter Venoso Central - CVC. [tese] Rio de Janeiro: Hospital Universitário Pedro Ernesto; 2014.

11. Azevedo LF, Sousa-Pinto B. Critical Appraisal of Economic Evaluation Studies (Part II): Cost-effectiveness and cost-utility analyses. Rev. Soc. Port. Anestesiol. [Internet], 2020 [cited 2020 Jul 18]; 29(1):18-21. DOI: https://doi.org/10.25751/rspa.19778

12. Richardson A, Melling A, Straughan C, Simms L, Coulter C, Elliot Y, et al. Central venous catheter dressing durability: an evaluation. J. Infect. Prev. [Internet], 2015 [cited 2020 May 3]; 16(6):256-61. DOI: https://doi.org/10.1177/1757177415594246

13. Pedrolo E, Danski MTR, Vayego AS, Oliveira GLR, Boostel R. Infection, local reaction and poor fixation of dressings for central venous cateter. Acta Paul. Enferm. [Internet], 2014 [cited 2020 Feb 17]; 27(1):63-8. DOI: https://doi.org/10.1590/19820194201400013

14. Gomes MLS, Aquino GAA, Barros LM, Queiroz PA, Oliveira FJG, Caetano JA. Assessment of short-term central venous catheter dressing practices. Rev. enferm. UERJ. [Internet], 2017 [cited 2020 Jul 10]; 25:e18196. DOI: http://dx.doi.org/10.12957/reuerj.2017.18196

15. Costa TC, Peregrino AAF, Rocha RG, Franco AS, Camerini F, Marta CB. Cost minimization analysis: use of dual and triple lumen central venous catheter. R. pesq.: cuid. fundam. online [Internet], 2020 [cited $2020 \mathrm{Jul} 10$ ]; 12:622-8. DOI: https://doi.org/10.9789/2175-5361.rpcfo.v12.8992 